\title{
On the Hölder continuous subsolution problem for the complex Monge-Ampère equation
}

\author{
Ngoc Cuong Nguyen ${ }^{1,2}$
}

Received: 23 March 2017 / Accepted: 8 December 2017 / Published online: 20 December 2017

(C) The Author(s) 2017. This article is an open access publication

\begin{abstract}
We give a necessary and sufficient condition for positive Borel measures such that the Dirichlet problem, with zero boundary data, for the complex Monge-Ampère equation admits Hölder continuous plurisubharmonic solutions. In particular, when the subsolution has finite Monge-Ampère total mass, we obtain an affirmative answer to a question of Zeriahi et al. (Complex Var. Elliptic Equ. 61(7):902-930, 2016).
\end{abstract}

Mathematics Subject Classification $53 \mathrm{C} 55 \cdot 35 \mathrm{~J} 96 \cdot 32 \mathrm{U} 40$

\section{Introduction}

Bedford and Taylor [3] proved the existence of plurisubharmonic solutions of the Dirichlet problem for the complex Monge-Ampère equation in a strictly pseudoconvex bounded domain $\Omega$ in $\mathbb{C}^{n}$. The solution is continuous or Hölder continuous provided that the data is continuous or Hölder continuous, respectively. In their subsequent fundamental paper [4] they developed pluripotential theory which becomes a powerful tool to study plurisubharmonic functions. Later, applying pluripotential theory methods the weak solution theory has been developed much further by Cegrell $[6,7]$ and Kołodziej $[18,19]$. The continuous solution is obtained for more general right hand sides in [19], in particular for $L^{p}$-density measures, $p>1$, as an important class. Recently, Guedj et al. [15] showed that the solution is Hölder

Communicated by N.Trudinger.

Dedicated to Professor Kang-Tae Kim on the occasion of his 60th birthday.

Ngoc Cuong Nguyen

Nguyen.Ngoc.Cuong@im.uj.edu.pl; cuongnn@postech.ac.kr

1 Faculty of Mathematics and Computer Science, Jagiellonian University, Łojasiewicza 6, 30-348 Kraków, Poland

2 Present Address: Department of Mathematics and Center for Geometry and its Applications, Pohang University of Science and Technology, Pohang 37673, Korea 
continuous, which is the optimal regularity, for such measures, under some extra assumptions. Finally, the extra assumptions were removed in [2], [8]. However, there is still an open question to find a characterisation for the measures admitting Hölder continuous solutions to the equation. If one requires only bounded solutions, then Kołodziej's subsolution theorem [16] gives such a criterion.

Let $\varphi \in P S H(\Omega) \cap C^{0, \alpha}(\bar{\Omega})$ with $0<\alpha \leq 1$, where $C^{0, \alpha}(\bar{\Omega})$ stands for the set of Hölder continuous functions of exponent $\alpha$ in $\bar{\Omega}$. Moreover, we assume that

$$
\varphi=0 \text { on } \partial \Omega \text {. }
$$

Given such a function $\varphi$ we consider the set of positive Borel measures on $\Omega$ which are dominated by the Monge-Ampère operator of $\varphi$, namely,

$$
\mathcal{M}(\varphi, \Omega):=\left\{\mu \text { is positive Borel measure: } \mu \leq\left(d d^{c} \varphi\right)^{n} \text { in } \Omega\right\} .
$$

For measures in this set we also say that $\varphi$ is a Hölder continuous subsolution to $\mu$. Let $\mu \in \mathcal{M}(\varphi, \Omega)$ and consider the Dirichlet problem for $0<\alpha^{\prime} \leq 1$,

$$
\begin{aligned}
& u \in P S H(\Omega) \cap C^{0, \alpha^{\prime}}(\bar{\Omega}) \\
& \left(d d^{c} u\right)^{n}=\mu, \\
& u=0 \text { on } \partial \Omega .
\end{aligned}
$$

The following problem [11, Question 17] was raised by Zeriahi.

Probelm 1.1 Can we always solve the Dirhichlet problem (1.3) for some $0<\alpha^{\prime} \leq 1$ ?

When $\varphi$ is merely bounded subsolution, the subsolution theorem in [16] has provided a unique bounded solution. Thus, to answer Zeriahi's question it remains to show the Hölder continuity of the bounded solution. Our main result is the following:

Theorem 1.2 Let $\mu \in \mathcal{M}(\varphi, \Omega)$. Assume that the Hölder continuous subsolution $\varphi$ has finite Monge-Ampère mass on $\Omega$, i.e.,

$$
\int_{\Omega}\left(d d^{c} \varphi\right)^{n}<+\infty
$$

Then, the Dirichlet problem (1.3) is solvable.

We actually obtain a necessary and sufficient condition for a measure in $\mathcal{M}(\varphi, \Omega)$ with finite total mass such that the Dirichlet problem (1.3) is solvable. Using this characterisation (Theorem 2.5) we can reprove the results from [2], [8], [15] in the case of zero boundary. We also show in Corollary 2.13 that there are several class of measures which satisfy the assumptions of the theorem. Charabati [9] has studied very recently Problem 1.1 for these measures.

There is a strong connection between Problem 1.1 and the Hölder continuity of weak solutions to Monge-Ampère equations on a compact Kähler manifold $(X, \omega)$. Namely, the characterisation [10, Theorem 4.3] (see also [20]) says that a positive Borel measure on $(X, \omega)$ is the Monge-Ampère measure of a Hölder continuous $\omega$-plurisubharmonic function if and only if it is dominated locally (on local coordinate charts) by the one of Hölder continuous plurisubharmonic functions. On the other hand, Dinh-Nguyen [12] has found another global characterisation for this problem by using super-potential theory [13]. This characterisation has been used in [22] to generalise the result obtained previously by Pham [21]. Our necessary and sufficient condition can be considered as the local analogue of [12]. It may possibly be used to give a local proof of the results in [21,22]. 


\section{A general characterisation}

Let $\Omega$ be a strictly pseudoconvex bounded domain in $\mathbb{C}^{n}$. Let $\rho \in C^{2}(\bar{\Omega})$ be a strictly plurisubhamonic defining function for $\Omega=\{\rho<0\}$. The standard Kähler form in $\mathbb{C}^{n}$ is denoted by $\beta:=d d^{c}|z|^{2}$. Without of loss generality we may assume that

$$
d d^{c} \rho \geq \beta \quad \text { on } \quad \bar{\Omega} .
$$

In this section we will prove a general characterisation of measures in $\mathcal{M}(\varphi, \Omega)$ which are Mong-Ampère measures of Hölder continuous plurisubharmonic functions. To state our result we need definitions and properties related to the Cegrell classes. The class $\mathcal{E}_{0}:=\mathcal{E}_{0}(\Omega)$ is defined by

$$
\left\{v \in P S H \cap L^{\infty}(\Omega): \lim _{z \rightarrow \partial \Omega} v(z)=0, \text { and } \int_{\Omega}\left(d d^{c} v\right)^{n}<+\infty\right\} .
$$

We will also work with a subclass of $\mathcal{E}_{0}$, namely,

$$
\mathcal{E}_{0}^{\prime}=\left\{v \in \mathcal{E}_{0}: \int_{\Omega}\left(d d^{c} v\right)^{n} \leq 1\right\} .
$$

The following basic properties of $\mathcal{E}_{0}$ and $\mathcal{E}_{0}^{\prime}$ which will be used later, and we refer the readers to $[6,7]$ for detailed proofs.

Proposition 2.1 We have

(a) The integration by parts holds true in $\mathcal{E}_{0}$; if $u, v \in \mathcal{E}_{0}$, so does $u+v$.

(b) Let $u \in \mathcal{E}_{0}$. Let $v \in P S H \cap L^{\infty}(\Omega)$ be such that $\lim _{z \rightarrow \partial \Omega} v(z)=0$, and $v \geq u$. Then, $v \in \mathcal{E}_{0}$ and

$$
\int_{\Omega}\left(d d^{c} v\right)^{n} \leq \int_{\Omega}\left(d d^{c} u\right)^{n}
$$

(c) Let $u, v \in \mathcal{E}_{0}^{\prime}$. Then, $\max \{u, v\} \in \mathcal{E}_{0}^{\prime}$ and $\max \{u,-s\} \in \mathcal{E}_{0}^{\prime}$ for every $s>0$.

(d) For $u_{1}, \ldots, u_{n} \in \mathcal{E}_{0}$ the Cegrell inequality reads as follows.

$$
\int_{\Omega} d d^{c} u_{1} \wedge \cdots \wedge d d^{c} u_{n} \leq\left(\int_{\Omega}\left(d d^{c} u_{1}\right)^{n}\right)^{\frac{1}{n}} \cdots\left(\int_{\Omega}\left(d d^{c} u_{n}\right)^{n}\right)^{\frac{1}{n}} .
$$

In what follows we always denote for $p>0$

$$
\|\cdot\|_{p}:=\left(\int_{\Omega}|\cdot|^{p} d V_{2 n}\right)^{\frac{1}{p}} \text { and }\|\cdot\|_{\infty}:=\sup _{\Omega}|\cdot|,
$$

where $d V_{2 n}$ is the Lebesgue measure in $\mathbb{C}^{n}$. The constant $C>0$ will appear in many places below, we understand that it is a uniform constant and it may differ from place to place.

Lemma 2.2 Let $v$ be a positive Borel measure on $\Omega$ and let $0<\alpha \leq 1$. Then,

$$
\left|\int_{\Omega}(u-v) d v\right| \leq C\|u-v\|_{1}^{\alpha} \quad \forall u, v \in \mathcal{E}_{0}^{\prime}
$$

if and only if

$$
\int_{\Omega}|u-v| d v \leq C\|u-v\|_{1}^{\alpha} \quad \forall u, v \in \mathcal{E}_{0}^{\prime} .
$$

Here, the constant $C>0$ is independent of $u, v$. 
Proof The sufficient condition is clear. To prove the necessary condition we first have that

$$
|u-v|=(\max \{u, v\}-u)+(\max \{u, v\}-v) .
$$

Since $u, v \in \mathcal{E}_{0}^{\prime}$, so $\max \{u, v\} \in \mathcal{E}_{0}^{\prime}$. Hence we apply (2.7) twice to get the desired inequality.

For a general positive Borel measure $v$ it defines a natural functional

$$
v(w):=\int_{\Omega} w d v
$$

for a measurable function $w$ in $\Omega$. We introduce the following notion which is probably a local counterpart of the one in Dinh-Sibony [13] and Dinh-Nguyen [12] for positive measures.

Definition 2.3 The measure $v$ is called to be Hölder (or $\alpha$-Hölder) continuous on $\mathcal{E}_{0}^{\prime}$ if there exists $0<\alpha \leq 1$ such that

$$
|v(u-v)|=\left|\int_{\Omega}(u-v) d v\right| \leq C\|u-v\|_{1}^{\alpha} \quad \forall u, v \in \mathcal{E}_{0}^{\prime},
$$

where the constant $C>0$ is independent of $u, v$.

Thanks to Lemma 2.2 we can verify the Hölder continuity of a positive Borel measure $v$ by using either (2.7) or (2.8). The following properties are consequences of the definition.

Lemma 2.4 Let $v$ be $\alpha$-Hölder continuous on $\mathcal{E}_{0}^{\prime}$, then we have

(a) $\mathcal{E}_{0} \subset L^{1}(d v)$;

(b) if $\mu \leq v$, then $\mu$ is also $\alpha$-Hölder continuous on $\mathcal{E}_{0}^{\prime}$;

(c) $v$ is a Radon measure, and $v$ vanishes on pluripolar sets in $\Omega$.

Proof The property $(a)$ is obvious. The property $(b)$ follows from (2.8). Lastly, since $C_{c}^{\infty}(\Omega) \subset \mathcal{E}_{0} \cap C^{0}(\bar{\Omega})-\mathcal{E}_{0} \cap C^{0}(\bar{\Omega})$ (see [7, Lemma 3.1]) it follows that $v$ is a Radon measure. Next, let $K \subset \Omega$ be a compact pluripolar set. Let $G_{j}$ be a decreasing sequence of compact sets in $\Omega$ satisfying

$$
G_{j+1} \subset \stackrel{o}{G_{j}} \text { and } \bigcap_{j=1}^{\infty} G_{j}=K .
$$

Let $h_{G_{j}}$ denote the relatively extremal function of $G_{j}$. By a theorem of Bedford-Taylor [4] we have

$$
\operatorname{cap}\left(G_{j}, \Omega\right)=\int_{\Omega}\left(d d^{c} h_{G_{j}}^{*}\right)^{n} \text { and } h_{G_{j}}^{*}=-1 \text { on } \stackrel{o}{G_{j}}
$$

Hence,

$$
\nu(K) \leq \int_{\Omega}\left|h_{G_{j}}^{*}\right| d \nu \leq C\left[\operatorname{cap}\left(G_{j}, \Omega\right)\right]^{\frac{1-\alpha}{n}}\left(\int_{\Omega}\left|h_{G_{j}}^{*}\right| d V_{2 n}\right)^{\alpha},
$$

where the second inequality used the fact that $v$ is $\alpha$-Hölder continuous on $\mathcal{E}_{0}^{\prime}$. Since $h_{G_{j}}^{*} \nearrow$ $h_{K}^{*} \equiv 0$ in $\Omega$, then the right hand side tends to 0 as $j \rightarrow \infty$. Thus, $v$ vanishes on pluripolar sets in $\Omega$.

Let us state the announced above characterisation for measures in $\mathcal{M}(\varphi, \Omega)$. 
Theorem 2.5 Let $\mu$ be a positive Borel measure such that $\mu \leq\left(d d^{c} \varphi\right)^{n}$ for $\varphi \in \operatorname{PSH}(\Omega) \cap$ $C^{0, \alpha}(\bar{\Omega})$ and $\varphi=0$ on $\partial \Omega$, where $0<\alpha \leq 1$. Assume that $\mu$ has finite total mass. Then, $\mu$ is Hölder continuous on $\mathcal{E}_{0}^{\prime}$ if and only if there exists $u \in P S H(\Omega) \cap C^{0, \alpha^{\prime}}(\bar{\Omega})$, where $0<\alpha^{\prime} \leq 1$, satisfying

$$
\left(d d^{c} u\right)^{n}=\mu, \quad u_{\mid \partial \Omega}=0 .
$$

Remark 2.6 One should remark that the Hölder exponent of $\mu$ on $\mathcal{E}_{0}^{\prime}$ is often different from the ones of $\varphi$ and $u$.

Let us prepare ingredients to prove the theorem. The following lemma will be its necessary condition.

Lemma 2.7 Let $\varphi \in \operatorname{PSH}(\Omega) \cap C^{0, \alpha}(\bar{\Omega})$ be such that $\varphi=0$ on $\partial \Omega$, where $0<\alpha \leq 1$. Assume that

$$
\int_{\Omega}\left(d d^{c} \varphi\right)^{n}<+\infty
$$

Then, the measure $v:=\left(d d^{c} \varphi\right)^{n}$ is Hölder continuous on $\mathcal{E}_{0}^{\prime}$.

Remark 2.8 On a compact Kähler manifold $(X, \omega)$ this is an analogue of Dinh-Nguyen [12, Proposition 4.1] for $\omega$-plurisubharmonic functions. The difference is that in the local setting one needs to deal with the boundary terms which do not appear in the compact manifold. We succeed to estimate these terms by using the Cegrell inequality (2.5).

Proof Denote $\beta=d d^{c}|z|^{2}$ and

$$
S_{k}:=\left(d d^{c} \varphi\right)^{k} \wedge \beta^{n-k} .
$$

Our goal is to show that there exists $0<\alpha \leq 1$ such that for $w, v \in \mathcal{E}_{0}^{\prime}$

$$
\int_{\Omega}|w-v| S_{n} \leq C\|w-v\|_{1}^{\alpha}
$$

We proceed by induction over $0 \leq k \leq n$. For $k=0$, the statement obviously holds true with $\alpha_{0}=1$. Assume that this holds for integers up to $k<n$. For simplicity let us denote

$$
S:=\left(d d^{c} \varphi\right)^{k} \wedge \beta^{n-k-1} .
$$

The induction hypothesis tells us that there is $0<\alpha_{k} \leq 1$ such that

$$
\int_{\Omega}|w-v| S \wedge \beta \leq C\|w-v\|_{1}^{\alpha_{k}} .
$$

We need to show that there exists $0<\alpha_{k+1} \leq 1$ such that

$$
\int_{\Omega}(w-v) d d^{c} \varphi \wedge S \leq C\|w-v\|_{1}^{\alpha_{k+1}}
$$

for every $v, w \in \mathcal{E}_{0}^{\prime}$ and $w \geq v$ (the general case will follow as in the proof of Lemma 2.2). Indeed, without loss of generality we may assume that

$$
\|w-v\|_{1}>0 .
$$

Otherwise, the inequality will follow directly from the induction step (2.10). Let us still write $\varphi$ to be the Hölder continuous extension of $\varphi$ onto a neighbourhood $U$ of $\bar{\Omega}$. Consider the convolution of $\varphi$ with the standard smooth kernel $\chi$, i.e., $\chi \in C_{c}^{\infty}(B(0,1))$ such that 
$\chi(z)=\chi(|z|) \geq 0$, supp $\chi \subset \subset B(0,1)$ and $\int_{\mathbb{C}^{n}} \chi(z) d V_{2 n}=1$. Then, we observe that for $z \in U$,

$$
\varphi_{\delta}(z)-\varphi(z)=\int_{B(0,1)}[\varphi(z-\delta \zeta)-\varphi(z)] \chi(\zeta) d V_{2 n}(\zeta) \leq C \delta^{\alpha}
$$

and

$$
\left|\frac{\partial^{2} \varphi_{\delta}}{\partial z_{j} \partial \bar{z}_{k}}(z)\right| \leq \frac{C\|\varphi\|_{\infty}}{\delta^{2}}
$$

We first have

$$
\begin{aligned}
\int_{\Omega}(w-v) d d^{c} \varphi \wedge S \leq & \left|\int_{\Omega}(w-v) d d^{c} \varphi_{\delta} \wedge S\right| \\
& +\left|\int_{\Omega}(w-v) d d^{c}\left(\varphi_{\delta}-\varphi\right) \wedge S\right| \\
& =: I_{1}+I_{2} .
\end{aligned}
$$

It follows from (2.10) and (2.13) that

$$
\begin{aligned}
I_{1} & \leq \frac{C\|\varphi\|_{\infty}}{\delta^{2}} \int_{\Omega}(w-v) S \wedge \beta \\
& \leq \frac{C\|\varphi\|_{\infty}}{\delta^{2}}\|w-v\|_{1}^{\alpha_{k}} .
\end{aligned}
$$

The integration by parts in $\mathcal{E}_{0}$ gives:

$$
\begin{aligned}
\int_{\Omega} w d d^{c}\left(\varphi_{\delta}-\varphi\right) \wedge S= & \int_{\Omega}\left(\varphi_{\delta}-\varphi\right) d d^{c} w \wedge S \\
& -\int_{\partial \Omega}\left(\varphi_{\delta}-\varphi\right) d^{c} w \wedge S .
\end{aligned}
$$

Notice that $d^{c} w \wedge S$ is a positive measure on $\partial \Omega$ with total mass is

$$
\int_{\Omega} d d^{c} w \wedge S<+\infty
$$

This finiteness of the integral is obtained as follow. By (2.1) it is clear that the defining function $\rho \in \mathcal{E}_{0}$ and $d d^{c} \rho \geq \beta$. Therefore

$$
\int_{\Omega} d d^{c} w \wedge S \leq \int_{\Omega} d d^{c} w \wedge\left(d d^{c} \varphi\right)^{k} \wedge\left(d d^{c} \rho\right)^{n-k-1} .
$$

The Cegrell inequality (2.5) gives us that the right hand side is less than

$$
\left(\int_{\Omega}\left(d d^{c} w\right)^{n}\right)^{\frac{1}{n}}\left(\int_{\Omega}\left(d d^{c} \varphi\right)^{n}\right)^{\frac{k}{n}}\left(\int_{\Omega}\left(d d^{c} \rho\right)^{n}\right)^{\frac{n-k-1}{n}} \leq C
$$

as $w \in \mathcal{E}_{0}^{\prime}$ and $\varphi \in \mathcal{E}_{0}$. Notice that here the constant $C$ is independent of $w$. Hence, using (2.12), we get that

$$
\left|\int_{\partial \Omega}\left(\varphi_{\delta}-\varphi\right) d^{c} w \wedge S\right| \leq C \delta^{\alpha} \int_{\Omega} d d^{c} w \wedge S
$$

Similarly, 


$$
\begin{gathered}
\int_{\Omega} d d^{c} v \wedge S<+\infty \\
\left|\int_{\partial \Omega}\left(\varphi_{\delta}-\varphi\right) d^{c} v \wedge S\right| \leq C \delta^{\alpha} \int_{\Omega} d d^{c} v \wedge S .
\end{gathered}
$$

Finally, using (2.12), (2.16), (2.17), (2.18), (2.19) and (2.20) we are able to finish the estimate

$$
\begin{aligned}
I_{2} \leq & \left|\int_{\Omega}\left(\varphi_{\delta}-\varphi\right) d d^{c} w \wedge S\right|+\left|\int_{\Omega}\left(\varphi_{\delta}-\varphi\right) d d^{c} v \wedge S\right| \\
& +\left|\int_{\partial \Omega}\left(\varphi_{\delta}-\varphi\right) d^{c} w \wedge S\right|+\left|\int_{\partial \Omega}\left(\varphi_{\delta}-\varphi\right) d^{c} v \wedge S\right| \\
\leq & C \delta^{\alpha}\left(\int_{\Omega} d d^{c} w \wedge S+\int_{\Omega} d d^{c} v \wedge S\right) \\
\leq & C \delta^{\alpha} .
\end{aligned}
$$

Thanks to (2.17) and (2.19) we have

$$
\begin{aligned}
\left|\int_{\Omega}(w-v) d d^{c} \varphi \wedge S\right| & \leq\left|\int_{\Omega} \varphi d d^{c} w \wedge S\right|+\left|\int_{\Omega} \varphi d d^{c} v \wedge S\right| \\
& \leq C\|\varphi\|_{\infty} .
\end{aligned}
$$

From this inequality we can assume that $0<\|w-v\|_{1}<1 / 100$. Combining (2.14), (2.15) and (2.21) we have

$$
\int_{\Omega}(w-v) d d^{c} \varphi \wedge S \leq \frac{C\|\varphi\|_{\infty}}{\delta^{2}}\|w-v\|_{1}^{\alpha_{k}}+C \delta^{\alpha} .
$$

Therefore, the proof of (2.11) is completed if we choose

$$
\delta=\|w-v\|_{1}^{\frac{\alpha_{k}}{3}}, \quad \alpha_{k+1}=\frac{\alpha \alpha_{k}}{3} .
$$

Thus, the lemma is proven.

The measures which are Hölder continuous on $\mathcal{E}_{0}^{\prime}$ satisfy the volume-capacity inequality, which is the content of the next proposition. This inequality plays a crucial role in deriving the apriori uniform estimate and stability estimates for the solutions to the Monge-Ampère equation.

Proposition 2.9 Assume that $v$ is $\alpha$-Hölder continuous on $\mathcal{E}_{0}^{\prime}$ with finite total mass on $\Omega$. Then, there exist uniform constants $\alpha_{1}>0$ and $C>0$ such that for every compact set $K \subset \Omega$

$$
v(K) \leq C \exp \left(\frac{-\alpha_{1}}{[\operatorname{cap}(K, \Omega)]^{\frac{1}{n}}}\right) .
$$

Proof First, we prove that for $v \in P S H \cap L^{\infty}(\Omega)$ and $\lim _{z \rightarrow \partial \Omega} v(z)=0$ satisfying $\int_{\Omega}\left(d d^{c} v\right)^{n} \leq 1$, there exist uniform constants $\alpha_{1}>0$ (small) and $C>0$ such that

$$
v(v<-s) \leq C e^{-\alpha_{1} s} \quad \forall s>0 .
$$

Indeed, put $v_{s}:=\max \{v,-s\}$. Then

$$
v(v<-s-1)=\int_{\{v<-s-1\}} d v \leq \int_{\Omega}\left|v_{s}-v\right| d \nu .
$$


Since $v_{s}, v \in \mathcal{E}_{0}^{\prime}$ and $v$ is $\alpha$-Hölder continuous on $\mathcal{E}_{0}^{\prime}$, the right hand side is bounded by

$$
C\left(\int_{\Omega}\left|v_{s}-v\right| d V_{2 n}\right)^{\alpha}
$$

Since $0 \leq v_{s}-v \leq \mathbf{1}_{\{v<-s\}}|v| \leq \mathbf{1}_{\{v<-s\}} e^{-\alpha_{2} v} / \alpha_{2}$ for $\alpha_{2}>0$ (to be determined later), we have

$$
\begin{aligned}
\int_{\Omega}\left|v_{s}-v\right| d V_{2 n} & \leq \frac{1}{\alpha_{2}} \int_{\{v<-s\}} e^{-\alpha_{2} v} d V_{2 n} \\
& \leq \frac{1}{\alpha_{2}} \int_{\Omega} e^{-\alpha_{2} v-\alpha_{2}(v+s)} d V_{2 n} \\
& \leq \frac{e^{-\alpha_{2} s}}{\alpha_{2}} \int_{\Omega} e^{-2 \alpha_{2} v} d V_{2 n}
\end{aligned}
$$

The last integral is uniformly bounded for $2 \alpha_{2}>0$ small enough thanks to [19, Lemma 4.1]. Combining (2.24), (2.25) and (2.26) we get that

$$
v(v<-s-1) \leq C e^{-\alpha \alpha_{2} s} .
$$

Hence, we obtained (2.23) for $\alpha_{1}=\alpha \alpha_{2}$ with $\alpha_{2}>0$ small enough.

To finish the proof of the proposition we use an argument which is inspired by the proofs in [1]. Let $K \subset \Omega$ be compact. Since $v$ vanishes on pluripolar sets (Lemma 2.4) we may assume that $K$ is non-pluripolar. Let $h_{K}^{*}$ be the relative extremal function of $K$ with respect to $\Omega$. Since $K \subset \Omega$ is compact, it is well-known that

$$
\lim _{\zeta \rightarrow \partial \Omega} h_{K}^{*}(\zeta)=0
$$

By [[4],Proposition 5.3] we have

$$
\tau^{n}:=\operatorname{cap}(K, \Omega)=\int_{\Omega}\left(d d^{c} h_{K}^{*}\right)^{n}>0 .
$$

Let $0<x<1$. Since the function $w:=\frac{h_{K}^{*}}{\tau}$ satisfies assumptions of the inequality (2.23), we have

$$
v\left(h_{K}^{*}<-1+x\right)=v\left(w<\frac{-1+x}{\tau}\right) \leq C \exp \left(-\frac{\alpha_{1}(1-x)}{\tau}\right) .
$$

Let $x \rightarrow 0^{+}$, we obtain

$$
v\left(h_{K}^{*} \leq-1\right) \leq C \exp \left(\frac{-\alpha_{1}}{[\operatorname{cap}(K, \Omega)]^{\frac{1}{n}}}\right) .
$$

Since $h_{K}=h_{K}^{*}$ outside a pluripolar set, we have

$$
v(K) \leq v\left(h_{K}=-1\right)=v\left(h_{K}^{*}=-1\right) \leq v\left(h_{K}^{*} \leq-1\right) .
$$

We combine (2.27) and (2.28) to finish the proof.

We can follow the proofs in [14], [15] and [17], to derive the stability estimate for the measures which are well dominated by capacity. 
Proposition 2.10 Suppose that $\mu=\left(d d^{c} u\right)^{n}$ for $u \in P S H(\Omega) \cap C^{0}(\bar{\Omega})$ and $\mu$ satisfies the inequality (2.22). Let $v \in P S H(\Omega) \cap C^{0}(\bar{\Omega})$ be such that $u=v$ on $\partial \Omega$. Then, there exist constants $C>0$ and $0<\alpha_{2}<1$ independent of $v$ such that

$$
\sup _{\Omega}(v-u) \leq C\left(\int_{\Omega} \max \{v-u, 0\} d \mu\right)^{\alpha_{2}} .
$$

Proof It readily follows from the one of [15, Theorem 1.1] with obvious adjustments.

We proceed to the proof of Theorem 2.5. Let $\mu \in \mathcal{M}(\varphi, \Omega)$ with $\mu(\Omega)<+\infty$. The theorem asserts that $\mu$ is Hölder continuous on $\mathcal{E}_{0}^{\prime}$ if and only there exists $u \in \operatorname{PSH}(\Omega) \cap$ $C^{0, \alpha^{\prime}}(\bar{\Omega}), 0<\alpha^{\prime} \leq 1$, solving

$$
\left(d d^{c} u\right)^{n}=\mu, \quad u_{\mid \partial \Omega}=0 .
$$

First, we see that the sufficient condition follows from Lemma 2.7 applied for $\varphi:=u$. It remains to prove the necessary condition. By Proposition 2.9, the measure $\mu$ satisfies the volume-capacity inequality (2.22). Therefore, using Kołodziej's result [19, Theorem 5.9] we solve $u \in \operatorname{PSH}(\Omega) \cap C^{0}(\bar{\Omega})$,

$$
\left(d d^{c} u\right)^{n}=\mu, \quad u_{\mid \partial \Omega}=0 .
$$

The existence of the Hölder continuous subsolution assures that the solution $u$ is Hölder continuous on the boundary. To see this, we get by the comparison principle [4] that

$$
\varphi \leq u \leq 0 \text { on } \Omega \text {. }
$$

Let us denote for $\delta>0$ small

$$
\Omega_{\delta}:=\{z \in \Omega: \operatorname{dist}(z, \partial \Omega)>\delta\} ;
$$

and for $z \in \Omega_{\delta}$ we define

$$
\begin{gathered}
u_{\delta}(z):=\sup _{|\zeta| \leq \delta} u(z+\zeta), \\
\hat{u}_{\delta}(z):=\frac{1}{\sigma_{2 n} \delta^{2 n}} \int_{|\zeta| \leq \delta} u(z+\zeta) d V_{2 n}(\zeta),
\end{gathered}
$$

where $\sigma_{2 n}$ is the volume of the unit ball.

Lemma 2.11 There exist constants $c_{0}=c_{0}(\varphi)>0$ and $\delta_{0}>0$ small such that for any $0<\delta<\delta_{0}$,

$$
u_{\delta}(z) \leq u(z)+c_{0} \delta^{\alpha}
$$

for every $z \in \partial \Omega_{\delta}$, where $\alpha$ is the Hölder exponent of $\varphi$.

Proof Fix a point $z \in \partial \Omega_{\delta}$. It follows from (2.31) and (2.33) that

$$
u_{\delta}(z)-u(z) \leq-\varphi(z) .
$$

Choose $\zeta_{0} \in \mathbb{C}^{n},\left\|\zeta_{0}\right\|=\delta$, such that $z+\zeta_{0} \in \partial \Omega$. Then, $\varphi\left(z+\zeta_{0}\right)=0$, and

$$
\begin{aligned}
-\varphi(z) & =\varphi\left(z+\zeta_{0}\right)-\varphi(z) \\
& \leq c_{0} \delta^{\alpha}
\end{aligned}
$$

for $0<\delta<\delta_{0}$ as $\varphi \in C^{0, \alpha}(\bar{\Omega})$. 
The following result follows essentially from [2, Theorem 3.3, Theorem 3.4].

Lemma 2.12 Fix $0<\alpha_{4}<1$. Then, we have for every $\delta>0$ small,

$$
\int_{\Omega_{\delta}}\left|\hat{u}_{\delta}-u\right| d V_{2 n} \leq C \delta^{\alpha_{4}} .
$$

Proof First, we know from the classical Jensen formula (see e.g [15, Lemma 4.3]) that

$$
\int_{\Omega_{2 \delta}}\left|\hat{u}_{\delta}-u\right| d V_{2 n} \leq C \delta^{2} \int_{\Omega_{\delta}} \Delta u(z) .
$$

Since both $\rho$ and $-\operatorname{dist}(z, \partial \Omega)$ are defining functions of the $C^{2}$ bounded domain, we have $-\rho(z) \geq c_{1} \operatorname{dist}(z, \partial \Omega)$ for some uniform constant $c_{1}>0$ depending on $\rho$ and $\Omega$. Hence,

$$
\Omega_{\delta} \subset\left\{\rho(z)<-c_{1} \delta\right\} .
$$

Put $2 \delta_{1}=c_{1} \delta$. Then,

$$
\int_{\Omega_{\delta}} \Delta u(z) \leq \int_{\left\{\rho<-2 \delta_{1}\right\}} \Delta u(z) .
$$

We may also assume that $\left\{\rho<-\delta_{1}\right\}$ has smooth boundary. Then, we have for a fixed $0<\alpha_{4}<1$,

$$
\begin{aligned}
& \int_{\left\{\rho<-2 \delta_{1}\right\}} \Delta u(z) \\
& \leq \delta_{1}^{-\left(2-\alpha_{4}\right)} \int_{\left\{\rho<-\delta_{1}\right\}}\left(-\rho-\delta_{1}\right)^{2-\alpha_{4}} \Delta u(z) \\
& =c_{n} \delta_{1}^{-\left(2-\alpha_{4}\right)} \int_{\left\{\rho<-\delta_{1}\right\}}\left(-\rho-\delta_{1}\right)^{2-\alpha_{4}} d d^{c} u \wedge \beta^{n-1} \\
& =c_{n} \delta_{1}^{-\left(2-\alpha_{4}\right)} \int_{\left\{\rho<-\delta_{1}\right\}} u d d^{c}\left(-\rho-\delta_{1}\right)^{2-\alpha_{4}} \wedge \beta^{n-1} \\
& \quad+c_{n} \delta_{1}^{-\left(2-\alpha_{4}\right)} \int_{\left\{\rho=-\delta_{1}\right\}}(-u) d^{c}\left(-\rho-\delta_{1}\right)^{2-\alpha_{4}} \wedge \beta^{n-1},
\end{aligned}
$$

where we used the integration by parts for the third equality, and $c_{n}$ is a constant depends only on $n$.

Next, we are going to get a uniform bound for the last two integrals in (2.39). We compute

$$
\begin{aligned}
d d^{c}\left(-\rho-\delta_{1}\right)^{2-\alpha_{4}}= & \left(2-\alpha_{4}\right)\left(1-\alpha_{4}\right)\left(-\rho-\delta_{1}\right)^{-\alpha_{4}} d \rho \wedge d^{c} \rho \\
& -\left(2-\alpha_{4}\right)\left(-\rho-\delta_{1}\right)^{1-\alpha_{4}} d d^{c} \rho .
\end{aligned}
$$

It is clear that

$$
\int_{\left\{\rho<-\delta_{1}\right\}}|u|\left(-\rho-\delta_{1}\right)^{-\alpha_{4}} d \rho \wedge d^{c} \rho \wedge \beta^{n-1} \leq C\|u\|_{\infty} \int_{\{\rho<0\}}|\rho|^{-\alpha_{4}} d V_{2 n} .
$$

The integral on the right hand converges as $0<\alpha_{4}<1$. Similarly,

$$
\int_{\left\{\rho<-\delta_{1}\right\}}|u|\left(-\rho-\delta_{1}\right)^{1-\alpha_{4}} d d^{c} \rho \wedge \beta^{n-1} \leq C\|u\|_{\infty}\|\rho\|_{\infty} \int_{\{\rho<0\}} d V_{2 n} .
$$

Therefore, by (2.40), (2.41) and (2.42) the first integral

$$
\int_{\left\{\rho<-\delta_{1}\right\}} u d d^{c}\left(-\rho-\delta_{1}\right)^{2-\alpha_{4}} \wedge \beta^{n-1} \leq C .
$$


We continue with the second one.

$$
\begin{aligned}
& \int_{\left\{\rho=-\delta_{1}\right\}}(-u) d^{c}\left(-\rho-\delta_{1}\right)^{2-\alpha_{4}} \wedge \beta^{n-1} \\
& \quad=\left(2-\alpha_{4}\right) \int_{\left\{\rho=-\delta_{1}\right\}}(-u)\left(-\rho-\delta_{1}\right)^{1-\alpha_{4}} d^{c} \rho \wedge \beta^{n-1} \\
& \leq C\|u\|_{\infty}\|\rho\|_{\infty} \int_{\left\{\rho=-\delta_{1}\right\}} d^{c} \rho \wedge \beta^{n-1} \\
& \leq C\|u\|_{\infty}\|\rho\|_{\infty} \int_{\{\rho<0\}} d d^{c} \rho \wedge \beta^{n-1} .
\end{aligned}
$$

Combining (2.38), (2.39), (2.43) (2.44), and then substituting $\delta=2 \delta_{1} / c_{1}$ we conclude that

$$
\int_{\Omega_{2 \delta}} \Delta u(z) \leq C \delta^{-\left(2-\alpha_{4}\right)} .
$$

Hence, using this and (2.37) we get that

$$
\begin{aligned}
\int_{\Omega_{\delta}}\left|u_{\delta}-u\right| d V_{2 n} & \leq \int_{\Omega_{2 \delta}}\left|u_{\delta}-u\right| d V_{2 n}+2\|u\|_{\infty} \int_{\Omega_{\delta} \backslash \Omega_{2 \delta}} d V_{2 n} \\
& \leq C \delta^{\alpha_{4}}+C \delta .
\end{aligned}
$$

We thus completed the proof of the lemma.

We are in the position to complete the theorem.

Proof (End of the proof of Theorem 2.5) It follows from Lemma 2.11 and $\hat{u}_{\delta} \leq u_{\delta}$ that

$$
\tilde{u}= \begin{cases}\max \left\{\hat{u}_{\delta}-c_{0} \delta^{\alpha}, u\right\} & \text { on } \Omega_{\delta}, \\ u & \text { on } \Omega \backslash \Omega_{\delta},\end{cases}
$$

belongs to $P S H(\Omega) \cap C^{0}(\bar{\Omega})$. Notice that

$$
\int_{\Omega}\left(d d^{c} \tilde{u}\right)^{n} \leq \int_{\Omega}\left(d d^{c} u\right)^{n}=\mu(\Omega)<+\infty .
$$

By applying Proposition 2.10 for $\tilde{u}$ and $u$, there is $0<\alpha_{2} \leq 1$ such that

$$
\begin{aligned}
\sup _{\Omega}(\tilde{u}-u) & \leq C\left(\int_{\Omega} \max \{\tilde{u}-u, 0\} d \mu\right)^{\alpha_{2}} \\
& \leq C\left(\int_{\Omega}|\tilde{u}-u| d \mu\right)^{\alpha_{2}} .
\end{aligned}
$$

Since $\mu \leq\left(d d^{c} \varphi\right)^{n}$, it follows from Lemma 2.7 that $\mu$ is Hölder continuous on $\mathcal{E}_{0}^{\prime}$. Moreover,

$$
\frac{u}{c_{2}}, \frac{\tilde{u}}{c_{2}} \in \mathcal{E}_{0}^{\prime},
$$

where $c_{2}=[\mu(\Omega)]^{1 / n}$. Therefore, there is $0<\alpha_{3} \leq 1$ such that

$$
\int_{\Omega}|\tilde{u}-u| d \mu \leq C[\mu(\Omega)]^{\frac{1-\alpha_{3}}{n}}\|\tilde{u}-u\|_{1}^{\alpha_{3}} .
$$

By Lemma 2.12 and (2.45) the right hand side is controlled by

$$
C \delta^{\alpha_{3} \alpha_{4}}
$$


for a fixed $0<\alpha_{4}<1$. Hence, using Lemma 2.11, (2.46) and (2.47),

$$
\begin{aligned}
\sup _{\Omega_{\delta}}\left(\hat{u}_{\delta}-u\right) & \leq \sup _{\Omega}(\tilde{u}-u)+c_{0} \delta^{\alpha} \\
& \leq C \delta^{\alpha_{5}},
\end{aligned}
$$

where $\alpha_{5}=\min \left\{\alpha, \alpha_{2} \alpha_{3} \alpha_{4}\right\}$. Thanks to [15, Lemma 4.2] we infer that

$$
\sup _{\Omega_{\delta}}\left(u_{\delta}-u\right) \leq C \delta^{\alpha_{5}}
$$

for $0<\delta<\delta_{0}$. Thus the proof of the Hölder continuity of $u$ in $\bar{\Omega}$ is completed.

Let us now complete the proof of the main theorem.

Proof of Theorem 1.2 Since $\mu \in \mathcal{M}(\varphi, \Omega)$ and $\int_{\Omega}\left(d d^{c} \varphi\right)^{n}<+\infty$, it follows from Lemma 2.4 and Lemma 2.7 that $\mu$ is Hölder continuous on $\mathcal{E}_{0}^{\prime}$. By Theorem 2.5 there exists $u \in \operatorname{PSH}(\Omega) \cap C^{0, \alpha^{\prime}}(\bar{\Omega}), 0<\alpha^{\prime} \leq 1$, solving

$$
\left(d d^{c} u\right)^{n}=d \mu, \quad u_{\mid \partial \Omega}=0 .
$$

Thus, the proof is completed.

Next, we give the following simple consequence of the main theorem. This result is also partially proved by another way in [9, Theorem 3.6].

Corollary 2.13 Let $\mu \in \mathcal{M}(\varphi, \Omega)$. Assume that $\mu$ has compact support in $\Omega$. Then Dirichlet problem (1.3) is solvable.

Proof Let $\rho$ be the defining function as in (2.1). Assume that $K:=\operatorname{supp} \mu \subset \subset \Omega$ and we fix a small constant $\delta>0$. There exists $A>0$ large enough such that $A \rho \leq \varphi-\delta$ in the neighbourhood $\Omega^{\prime} \subset \subset \Omega$ of $K$. Set

$$
\tilde{\varphi}(z)=\max \{\varphi(z)-\delta, A \rho(z)\} .
$$

We easily see that $\tilde{\varphi}=\varphi-\delta$ on $\Omega^{\prime}$ and $\tilde{\varphi}=A \rho$ near the boundary $\partial \Omega$, and $\tilde{\varphi}$ is Hölder continuous in $\bar{\Omega}$ with the same exponent of $\varphi$. Therefore,

$$
\mu \leq\left(d d^{c} \tilde{\varphi}\right)^{n} \text { and } \int_{\Omega}\left(d d^{c} \tilde{\varphi}\right)^{n}<+\infty .
$$

The finiteness of the integral is followed from the Chern-Levine-Nirenberg inequality and $C^{2}$-smoothness near the boundary of $\tilde{\varphi}$. Using Theorem 1.2 we can solve the Dirichlet problem (1.3) for $\mu$.

It is obvious that the Lebesgue measure $d V_{2 n}$ is 1-Hölder continuous on $\mathcal{E}_{0}^{\prime}$. Then, we have the following general result which contains previous results in [2], [8], [15] for the zero boundary case.

Corollary 2.14 Let $v$ be Hölder continuous on $\mathcal{E}_{0}^{\prime}$. Let $0 \leq f \in L^{p}(\Omega, d v), p>1$. Then, $\mu:=f d v$ is Hölder continuous on $\mathcal{E}_{0}^{\prime}$. In particular, if $\mu \in \mathcal{M}(\varphi, \Omega)$, the Dirichlet problem (1.3) admits a unique solution. 
Proof The case $n=1$ is classical, so we assume that $n \geq 2$. It is sufficient to show that $f d v$ is Hölder continuous on $\mathcal{E}_{0}^{\prime}$. Assume that $u, v \in \mathcal{E}_{0}^{\prime}$ and $u \geq v$. Consider the triples

$$
\left(q_{1}, q_{2}, q_{3}\right)=\left((n-1) p+1, \frac{(n-1) p+1}{n-1}, \frac{(n-1) p+1}{(n-1)(p-1)}\right)
$$

and

$$
\left(\left[(u-v)^{n} f\right]^{\frac{1}{q_{1}}}, f^{\frac{p}{q_{2}}},(u-v)^{\frac{1}{q_{3}}}\right) .
$$

Then, the generalised Hölder inequality for

$$
\frac{1}{q_{1}}+\frac{1}{q_{2}}+\frac{1}{q_{3}}=1
$$

gives us that

$$
\int_{\Omega}(u-v) f d v \leq\left(\int_{\Omega}(u-v)^{n} f d v\right)^{1 / q_{1}}\|f\|_{L^{p}(d v)}^{p / q_{2}}\|u-v\|_{L^{1}(d v)}^{1 / q_{3}} .
$$

Since $v$ is Hölder continuous on $\mathcal{E}_{0}^{\prime}$, there is $0<\alpha \leq 1$ such that

$$
\int_{\Omega}|u-v| d v \leq\|u-v\|_{1}^{\alpha}
$$

Therefore, to end the proof, we need to verify that the first factor of the right hand side in (2.48) is uniformly bounded. Indeed, by the Hölder inequality we have

$$
\int_{K} f d v \leq\|f\|_{L^{p}(d v)}^{\frac{1}{p}}[v(K)]^{\frac{1}{q}},
$$

where $1 / p+1 / q=1$. Hence, $f d v$ satisfies the volume-capacity inequality (2.22). By Kołodziej's theorem [19, Theorem 5.9] there exists $\psi \in P S H(\Omega) \cap C^{0}(\bar{\Omega})$ satisfying $\left(d d^{c} \psi\right)^{n}=f d \nu, \quad \psi_{\left.\right|_{\partial \Omega}}=0$ and

$$
\|\psi\|_{\infty} \leq C\left(\|f\|_{L^{p}(d v)}, \Omega, v\right) .
$$

By an estimate of Błocki [5] we have

$$
\int_{\Omega}(u-v)^{n}\left(d d^{c} \psi\right)^{n} \leq n !\|\psi\|_{\infty}^{n} \int_{\Omega}\left(d d^{c} v\right)^{n} .
$$

The right hand side under controlled as $v \in \mathcal{E}_{0}^{\prime}$. It follows from (2.48), (2.49), (2.50) and (2.51) that

$$
\int_{\Omega}(u-v) f d v \leq C\|u-v\|_{1}^{\frac{\alpha}{q_{3}}}
$$

Thus, the corollary follows.

Finally, we emphasise that several interesting examples of positive Borel measures for which one can solve the Dirichlet problem (1.3) are given in Charabati [9] (see also [21], [22]). In particular, the class of Hausdorff-Riesz measures of order $2 n-2+\varepsilon$ with $0<\varepsilon \leq 2$ in $\Omega$. By Theorem 2.5 such measures are Hölder continuous on $\mathcal{E}_{0}^{\prime}$. On the other hand we can prove directly this result. 
Lemma 2.15 Let $\mu$ be a Hausdorff-Riesz, measure of order $2 n-2+\varepsilon$ with $0<\varepsilon \leq 2$ in $\Omega$, i.e., for every $B(z, \delta) \subset \Omega$

$$
\mu(B(z, \delta)) \leq C \delta^{2 n-2+\varepsilon},
$$

where $0<\delta<\delta_{0}$ small. Assume that $\mu$ has finite total mass. Then, $\mu$ is Hölder continuous on $\mathcal{E}_{0}^{\prime}$.

Proof By classical potential theory we solve $\varphi \in S H(\Omega) \cap C^{0, \alpha}(\bar{\Omega})$ satisfying

$$
\Delta \varphi=d \mu, \quad \varphi_{\left.\right|_{\partial \Omega}}=0 .
$$

The Hölder continuity follows from (2.52) (see e.g. [10, Remark 4.2]). Let us still write $w$ for its Hölder continuous extension onto a neighbourhood $U$ of $\bar{\Omega}$. Let $w, v \in \mathcal{E}_{0}^{\prime}$ and $w \geq v$. We need to show that

$$
\int_{\Omega}(w-v) \Delta \varphi \leq C\|w-v\|_{1}^{\alpha^{\prime}}
$$

for some $0<\alpha^{\prime} \leq 1$. We can write $\Delta \varphi=n d d^{c} \varphi \wedge \beta^{n-1}$ in the sense of measures. Define $\varphi_{\delta}$ is the convolution of $\varphi$ with the standard smooth kernel, then we have as in (2.12) and (2.13) that

$$
\begin{gathered}
\left|\varphi_{\delta}(z)-\varphi(z)\right| \leq C \delta^{\alpha}, \\
\left|\Delta \varphi_{\delta}(z)\right| \leq \frac{C\|\varphi\|_{\infty}}{\delta^{2}} .
\end{gathered}
$$

From this point the proof goes the same as the one in Lemma 2.7 with the closed positive current $S=\beta^{n-1}$.

Acknowledgements I am very grateful to Sławomir Kołodziej for giving many valuable comments on the drafts of the paper which helped to improve significantly its final version. The author is supported by the NRF Grant 2011-0030044 (SRC-GAIA) of The Republic of Korea.

Open Access This article is distributed under the terms of the Creative Commons Attribution 4.0 International License (http://creativecommons.org/licenses/by/4.0/), which permits unrestricted use, distribution, and reproduction in any medium, provided you give appropriate credit to the original author(s) and the source, provide a link to the Creative Commons license, and indicate if changes were made.

\section{References}

1. Åhag, P., Cegrell, U., Kołodziej, S., Pham, H.H., Zeriahi, A.: Partial pluricomplex energy and integrability exponents of plurisubharmonic functions. Adv. Math. 222, 2036-2058 (2009)

2. Baracco, L., Khanh, T.-V., Pinton, S., Zampieri, G.: Hölder regularity of the solution to the complex Monge-Ampère equation with $L^{p}$ density. Calc. Var. Partial Differ. Equ. 55(4), p 8 (2016), Art 74

3. Bedford, E., Taylor, B.A.: The Dirichlet problem for a complex Monge-Ampère operator. Invent. Math. 37, 1-44 (1976)

4. Bedford, E., Taylor, B.A.: A new capacity for plurisubharmonic functions. Acta Math. 149, 1-40 (1982)

5. Błocki, Z.: Estimates for the complex Monge-Ampère operator. Bull. Pol. Acad. Sci. Math. 41(2), 151157 (1993)

6. Cegrell, U.: Pluricomplex energy. Acta Math. 180(2), 187-217 (1998)

7. Cegrell, U.: The general definition of the complex Monge-Ampère operator. Ann. Inst. Fourier (Grenoble) 54, 159-179 (2004)

8. Charabati, M.: Hölder regularity for solutions to complex Monge-Ampère equations. Ann. Polon. Math. 113(2), 109-127 (2015)

9. Charabati, M.: Regularity of solutions to the Dirichlet problem for Monge-Ampère equations. Preprint, arXiv: 1511.01858 
10. Demailly, J.-P., Dinew, S., Guedj, V., Kołodziej, S., Pham, H.-H., Zeriahi, A.: Hölder continuous solutions to Monge-Ampère equations. J. Eur. Math. Soc. (JEMS) 16(4), 619-647 (2014)

11. Dinew, S., Guedj, V., Zeriahi, A.: Open problems in pluripotential theory. Complex Var. Elliptic Equ. 61(7), 902-930 (2016)

12. Dinh, T.-C., Nguyen, V.-A.: Characterization of Monge-Ampère measures with Hölder continuous potentials. J. Funct. Anal. 266(1), 67-84 (2014)

13. Dinh, T.-C., Sibony, N.: Super-potentials of positive closed currents, intersection theory and dynamics. Acta Math. 203(1), 1-82 (2009)

14. Eyssidieux, P., Guedj, V., Zeriahi, A.: Singular Kähler-Einstein matrics. J. AMS 22, 607-639 (2009)

15. Guedj, V., Kołodziej, S., Zeriahi, A.: Hölder continuous solutions to Monge-Ampère equations. Bull. Lond. Math. Soc. 40, 1070-1080 (2008)

16. Kołodziej, S.: The range of the complex Monge-Ampère operator. II. Indiana Univ. Math. J. 44(3), 765-782 (1995)

17. Kołodziej, S.: Some sufficient conditions for solvability of the Dirichlet problem for the complex MongeAmpère operator. Ann. Polon. Math. 65, 11-21 (1996)

18. Kołodziej, S.: The complex Monge-Ampère equation. Acta Math. 180, 69-117 (1998)

19. Kołodziej, S.: The complex Monge-Ampère equation and pluripotential theory. Memoirs Am. Math. Soc. 178, 64 (2005)

20. Kołodziej, S.: Hölder continuity of solutions to the complex Monge-Ampère equation with the right hand side in $L^{p}$. The case of compact Kähler manifolds. Math. Ann. 342, 379-386 (2008)

21. Pham, H.-H.: Hölder continuity of solutions to the Monge-Ampère equations on compact Kähler manifolds. Ann. Inst. Fourier (Grenoble) 60(5), 1857-1869 (2010)

22. Vu, D.V.: Complex Monge-Ampère equation for measures supported on real submanifolds. Preprint, arXiv:1608.02794 (to appear in Math. Ann) 